\title{
Sex differences in the prediction of the effectiveness of paroxetine for patients with major depressive disorder identified using a receiver operating characteristic curve analysis for early response [Corrigendum]
}

\author{
Tomita T, Norio YF, Sato Y, et al. Neuropsychiatr Dis Treat. 2014;10:599-606. \\ On page 599, there is an error in the author list "Yasui-Furukori Norio" should read "Norio Yasui-Furukori".
}

